Notfall Rettungsmed 2023 $26: 114-120$ https://doi.org/10.1007/s10049-021-00923-8 Angenommen: 18. Juni 2021 Online publiziert: 9. Juli 2021

(c) Der/die Autor(en) 2021

\section{Allgemeinmedizin in der Notaufnahme - Welche Patienten? Welche Beschwerden?}

\author{
Tanja Schleef · Nils Schneider · Olaf Krause \\ Institut für Allgemeinmedizin, Medizinische Hochschule Hannover, Hannover, Deutschland
}

\title{
Zusammenfassung
}

Hintergrund: Vor dem Hintergrund des zunehmenden Anteils ambulant verbleibender Patienten wurde die Fachdisziplin Allgemeinmedizin in die zentrale Notaufnahme (ZNA) einer Universitätsklinik integriert. In der ZNA werden der Allgemeinmedizin diejenigen Patienten zugewiesen, die sich mit Beratungsanlässen aus dem allgemeinmedizinischen Spektrum vorstellen sowie bei Ersteinschätzung eine niedrige Behandlungsdringlichkeit aufweisen. Ziel war es, diese Patienten hinsichtlich ihrer Beratungsanlässe zu charakterisieren.

Methodik: Deskriptive Auswertung auf Basis der klinischen Routinedaten sowie eines selbst entwickelten Erhebungsbogens.

Ergebnisse: Etwa $80 \%$ der Patienten suchten die Notaufnahme ohne ärztliche Einweisung auf. Die fünf häufigsten Beratungsanlässe waren Bauchschmerzen, Rückenschmerzen, Brustschmerzen, Schmerzen der unteren Extremitäten sowie Übelkeit und/oder Erbrechen. Bei knapp $25 \%$ der Patienten setzten die Beschwerden erst am Tag der Vorstellung ein, bei etwa $33 \%$ bestanden diese seit mehr als einer Woche. Die Hälfte der Patienten war wegen der Beschwerden bereits in ambulanter ärztlicher Behandlung, bei Patienten mit einer Beschwerdedauer von länger als einer Woche lag dieser Anteil bei mehr als $80 \%$. Patienten mit den Beratungsanlässen Fieber oder Verschlechterung des Allgemeinzustands wurden anteilig am häufigsten stationär aufgenommen. Von den Selbstzuweisern konnten knapp $94 \%$ in die ambulante Versorgung entlassen werden.

Diskussion: Patienten suchen auch mit nichtakuten Beschwerden und trotz vorangegangener ambulanter ärztlicher Behandlung die Notaufnahme auf. Die häufigsten Beratungsanlässe allgemeinmedizinisch versorgter Patienten in der ZNA decken sich nur zum Teil mit denen der hausärztlichen Versorgung.

\section{Schlüsselwörter}

Universitätsklinik · Ambulante Versorgung · Beratungsanlass · Selbstzuweiser · Versorgungsforschung

Notaufnahmen haben seit Jahren ein steigendes Patientenaufkommen zu verzeichnen. Sie werden zunehmend durch Patienten mit niedriger Behandlungsdringlichkeit in Anspruch genommen, die nach der Notfallbehandlung in die ambulante Regelversorgung entlassen werden. Die Integration der Fachdisziplin Allgemeinmedizin in die Notaufnahme stellt eine Möglichkeit dar, auf die Versorgungsrealität mit steigendem Anteil ambulanter Behandlungsfälle zu reagieren.
Notaufnahmen haben kontinuierlich steigende Patientenzahlen zu verzeichnen, die jährlichen Zuwachsraten betragen für Deutschland bis zu $9 \%[4,12,25]$. Insgesamt suchen mehr als $20 \mathrm{Mio}$. Patienten pro Jahr die Notaufnahmen auf $[14,20]$. Eigentlich sind Notaufnahmen zur Behandlung schwer kranker Patienten inklusive Schockraummanagement mit weiterer stationärer Versorgung gedacht [11]. Mittlerweile machen jedoch Selbstzuweiser („walk-in emergencies") einen beträchtlichen und meist mehrheitlichen Anteil des Patientenaufkommens aus [4, 
6, 20]. Nach Diagnostik und Behandlung, soweit diese im Rahmen der Notfallerstversorgung erforderlich war, kann mehr als die Hälfte aller vorstelligen Patienten in die ambulante Regelversorgung entlassen werden $[3,14,20]$.

Die Gründe für diesen großen und weiter zunehmenden Anteil ambulanter Behandlungsfälle sind vielschichtig und in veränderten Patientenpräferenzen, Schwierigkeiten im Zugang zur ambulanten Versorgung und in erwarteten Qualitätsvorteilen durch die interdisziplinären Behandlungsmöglichkeiten eines Krankenhauses zu finden [2, 15, 19, 23]. Ebenso tragen mangelndes Einschätzungsvermögen bezüglich der Dringlichkeit der eigenen Beschwerden sowie die Unkenntnis alternativer ambulanter Behandlungsstrukturen zum Anstieg ambulanter Behandlungsfälle in Notaufnahmen bei [19, 21, 23]. Es wird angenommen, dass ein Teil dieser ambulant versorgten Patienten auch adäquat während der üblichen Sprechzeiten in den Arztpraxen bzw. außerhalb der Sprechzeit durch den ärztlichen Bereitschaftsdienst behandelt werden könnte [4].

Als Reaktion auf die Zunahme ambulanter Patienten in der zentralen Notaufnahme (ZNA) hat die Medizinische Hochschule Hannover (MHH) Allgemeinärzte (Fachärzte für Allgemeinmedizin oder hausärztlich tätige Internisten) in die ZNA integriert. Die Allgemeinärzte sind in einem von der Kassenärztlichen Vereinigung Niedersachsen unterstützten Modellprojekt am Institut für Allgemeinmedizin der $\mathrm{MHH}$ angestellt, ihre Tätigkeit in der ZNA erfolgt rechtlich und organisatorisch als angestellte Klinikärzte und nicht freiberuflich oder im Rahmen der vertragsärztlichen Tätigkeit (kein Kassenarztsitz, keine Bereitschaftsdienstpraxis; [17]). Die allgemeinmedizinische Patientenversorgung in der Notaufnahme wird seit 2014 regelmäßig an den Werktagen von 10 bis $18 \mathrm{Uhr}$ angeboten. Ziel ist eine allgemeinärztlich orientierte Diagnostik und Erstversorgung von Patienten mit weniger dringlichen Beschwerden. Dies umfasst die Leistungen im Rahmen der gesetzlich definierten Notfallerstversorgung einschließlich der Abklärung einer stationären Behandlungsindikation sowie - bei fehlender Indikation zur stationären Aufnahme - die Rückführung in die haus- ärztliche Regelversorgung. Der Allgemeinmedizin stehen hierbei für die notwendigen Leistungen zur Erstversorgung die gleichen Ressourcen des Krankenhauses wie den anderen Fachdisziplinen der ZNA zur Verfügung. Die Zuordnung der Patienten zu einer Fachdisziplin innerhalb der ZNA erfolgt durch erfahrene Pflegekräfte auf Basis der klinischen Einschätzung der Leitsymptome, wobei Patienten mit nachrangiger Behandlungsdringlichkeit sowie typischen allgemeinmedizinischen Beratungsanlässen (z. B. Husten, Bauchschmerzen, Rückenschmerzen) der Allgemeinmedizin zugewiesen werden.

Nichtdringliche Patienten in Notaufnahmen waren inzwischen Gegenstand mehrerer wissenschaftlicher Untersuchungen. In der Regel findet jedoch keine Unterscheidung zwischen Patienten mit traumatischen und nichttraumatischen Beratungsanlässen statt, die Patienten werden zumeist - unabhängig von der versorgenden Fachdisziplin - als Gesamtkollektiv betrachtet $[13,15,19,23]$. Erste Untersuchungen zu spezifischen Beratungsanlässen existieren im Rahmen der Erhebungen des EMANet für Patienten mit respiratorischen Beschwerden [5]. Differenzierte Betrachtungen nichtdringlicher Patienten in Notaufnahmen mit Blick auf das gesamte Spektrum allgemeinmedizinischer Beratungsanlässe fehlen jedoch bislang für Deutschland, die feste Integration der Allgemeinmedizin in Krankenhausnotaufnahmen ist in Deutschland ebenfalls nicht üblich.

Ziel dieser Arbeit ist es, Patienten mit geringer Behandlungsdringlichkeit, die in einer universitären Notaufnahme allgemeinmedizinisch versorgt werden, am Beispiel der $\mathrm{MHH}$ differenziert zu beschreiben.

\section{Fragestellung}

- Mit welchen Beratungsanlässen werden diese Patienten in der Notaufnahme vorstellig?

- Wie lange bestanden diese Beschwerden bereits und wurde vor der Vorstellung aufgrund dieser Beschwerden ärztliche Hilfe in Anspruch genommen?

- Wohin verlassen die Patienten die Notaufnahme (ambulant/stationär) je nach Beratungsanlass?

\section{Methoden}

Die Erhebung der allgemeinärztlichen Patientenkontakte erfolgte über die Routinedaten der ZNA im Klinikinformationssystem. Die der Allgemeinmedizin zugeordneten Patienten bildeten jedoch nur einen Teil der allgemeinärztlichen Tätigkeit in der ZNA ab. Initial allgemeinmedizinische Patienten, die im Versorgungsverlauf an eine andere Fachdisziplin abgegeben wurden, weil beispielsweise aufgrund des Krankheitsbilds eine Übernahme durch eine andere Fachrichtung bzw. eine stationäre Aufnahme veranlasst wurde, wurden administrativ nicht unter Allgemeinmedizin erfasst. Um Informationen zu diesen Patienten zu erhalten und um die Routinedaten zu ergänzen, erfolgte durch den diensthabenden Allgemeinarzt eine strukturierte Kurzdokumentation mittels eines zusätzlichen Erhebungsbogens über einen Zeitraum von 18 Monaten. Die Beratungsanlässe wurden als Freitexte dokumentiert und anschließend in Kategorien überführt, die sich an der International Classification of Primary Care (ICPC-2; [8]) bzw. ihrer für die Notaufnahme angepassten kürzeren Fassung [9] orientierten.

Die erhobenen Daten wurden mit IBM SPSS Statistics 26 deskriptiv ausgewertet. Die Speicherung und Verarbeitung aller personenbezogenen Daten erfolgte pseudonym über die Fall- und Patientennummer.

Die Untersuchung ist Teil der Begleitevaluation zur Integration der Allgemeinmedizin in die zentrale Notaufnahme der $\mathrm{MHH}$, für die ein positives Ethikvotum der Ethikkommission der Medizinischen Hochschule Hannover (Nr. 2067-2013) vorliegt.

\section{Ergebnisse}

Zwischen 01.01.2018 und 30.06.2019 wurden 2469 Patienten (davon 55,0\% weiblich) in der ZNA der $\mathrm{MHH}$ durch einen Allgemeinarzt versorgt, was etwa einem Drittel der internistischen Notfälle im Vergleichszeitraum (Montag bis Freitag, jeweils 10-18 Uhr) entspricht. Das durchschnittliche Patientenalter lag bei 46,2 Jahren (Altersspanne 14-100 Jahre), die Altersverteilung ist in $\bullet$ Abb. 1 dargestellt. 


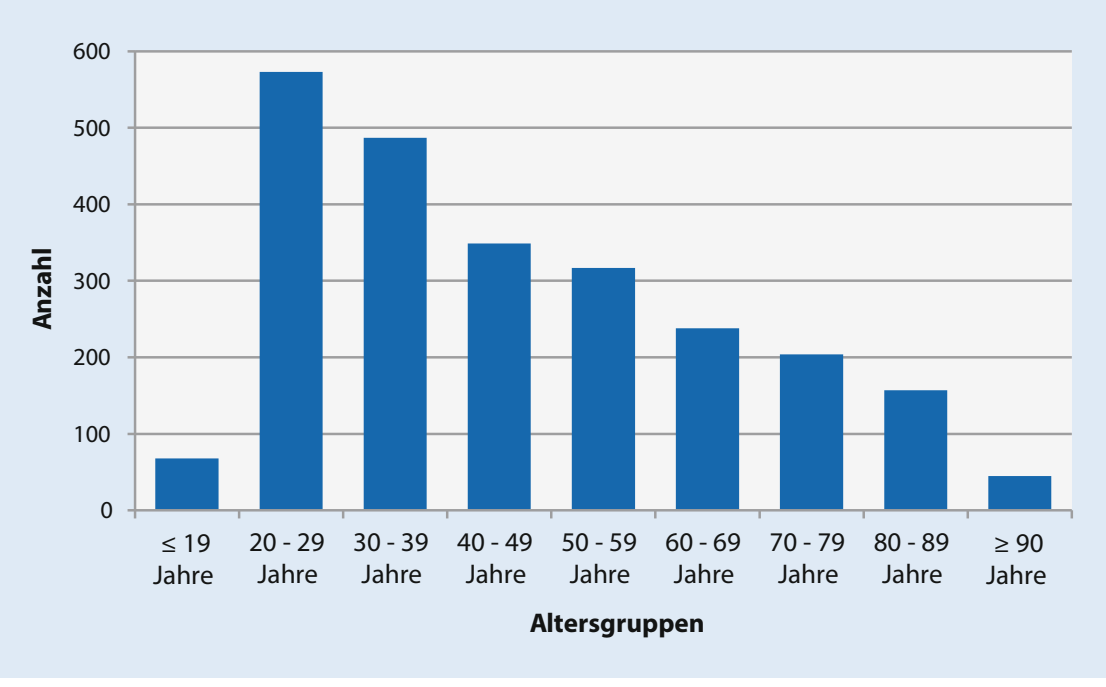

Abb. 1 A Altersverteilung der allgemeinmedizinisch versorgten ZNA-Patienten

Art der Einweisung, Beschwerdedauer und vorangegangene Arztkontakte

Für 1942 Patienten lagen vollständige Angaben zur Art der Einweisung sowie zur Dauer der gesundheitlichen Beschwerden vor. 349 Patienten $(18,0 \%)$ stellten sich mit einer ärztlichen Einweisung in der ZNA vor, $1557(80,2 \%)$ kamen als Selbstzuweiser in die Notaufnahme, 35 Patienten (1,8\%) waren über die ZNA wiedereinbestellt worden und ein Patient wurde über eine Hochschulambulanz an die ZNA zur Vorstellung verwiesen.

481 Patienten (24,8\%) wiesen akute Beschwerden auf, die erst am Tag der Vorstellung auftraten, entsprechend bestanden bei knapp drei Viertel $(74,6 \%)$ der Patienten die Beschwerden seit mindestens dem Vortag. Bei 654 Patienten $(33,6 \%)$ traten die Beschwerden vor mehr als einer Woche auf, bei 531 (27,3\%) dieser Patienten bestanden die Beschwerden bereits seit mehr als zwei Wochen.

Inklusive der Patienten mit ärztlicher Einweisung gaben 983 Patienten (50,6\%) an, vor Aufsuchen der ZNA wegen der aktuellen Beschwerden bereits in ambulanter Behandlung gewesen zu sein. Mit zunehmender Beschwerdedauer stieg der Anteil der Patienten mit vorausgegangenen Behandlungen von $18,1 \%(n=85)$ in der Gruppe mit akuten Beschwerden bis auf $81,3 \%(n=520)$ in der Gruppe mit einer Beschwerdedauer von länger als einer Woche (• Abb. 2).
Von den Selbstzuweisern hatten 601 $(38,6 \%)$ vor dem Aufsuchen der ZNA mindestens einen ambulanten Arztkontakt. Davon fanden 466 Arztkontakte mit Hausärzten und 190 Arztkontakte mit Fachspezialisten statt. 30 Patienten waren beim ärztlichen Bereitschaftsdienst, 101 Patienten in einer Notaufnahme vorstellig. Für 33 wiedereinbestellte Patienten wurden ebenfalls ambulante Arztkontakte dokumentiert, zu 44 Selbstzuweisern sowie zu zwei wiedereinbestellten Patienten lagen keine Angaben hinsichtlich etwaiger vorangegangener Arztkontakte vor.

\section{Beratungsanlässe}

Für 2107 Patienten wurden 2364 Beratungsanlässe dokumentiert, insgesamt wurden 106 verschiedene Beratungsanlässe erfasst. Die häufigsten Beratungsanlässe waren Bauchschmerzen (318/13,5\%), Rückenschmerzen (219/9,3\%), Brustschmerzen $(121 / 5,1 \%)$, Schmerzen der unteren Extremitäten/Hüftschmerzen (100/4,2\%) sowie Übelkeit und/oder Erbrechen $(94 / 4,0 \%)$ (• Tab. 1). Die 20 häufigsten Beratungsanlässe decken $72,0 \%$ aller Beratungsanlässe der allgemeinmedizinisch versorgten Patienten ab.

Das durchschnittliche Patientenalter variierte mit dem Beratungsanlass. Unter den 20 häufigsten Beratungsanlässen waren diejenigen Patienten am jüngsten, die sich mit Übelkeit und/oder Erbrechen vorstellten (38,0 Jahre), gefolgt von Patienten mit Durchfall (38,8 Jahre). Das höchste Durchschnittsalter fand sich bei Patienten mit einer Verschlechterung des Allgemeinzustands (64,9 Jahre), das zweithöchste bei Patienten mit Hypertonie (58,8 Jahre). Geschlechtsspezifisch zeigte sich der höchste Anteil an Frauen beim Beratungsanlass Hypertonie (66,2\%), während Männer beim Beratungsanlass Fieber dominierten $(59,6 \%)$.

Abhängig vom Beratungsanlass fanden sich Unterschiede im Anteil der Patienten mit vorangegangenen ambulanten Arztkontakten. Dieser war am höchsten für Patienten, die zum Ausschluss einer Thrombose oder wegen Verschlechterung ihres Allgemeinzustands (66,7\% bzw. 59,5\%) in die Notaufnahme kamen, zugleich war in beiden Patientengruppen auch der Anteil an ärztlichen Einweisungen am höchsten (47,0\% bzw. 34,0\%). Patienten, die zur Abklärung einer Synkope oder mit Kreislaufbeschwerden vorstellig wurden, hatten anteilig die geringsten vorangegangenen Arztkontakte (26,4\% bzw. $28,5 \%$ ) sowie den höchsten Anteil an akuten, am Tag der Vorstellung aufgetretenen Beschwerden $(77,0 \%$ bzw. 82,9\%). Der niedrigste Anteil an akuten Beschwerden fand sich bei Patienten mit Schmerzen der unteren Extremitäten/Hüftschmerzen oder mit Husten (5,0\% bzw. 5,6\%). Die Mehrheit der Patienten mit Luftnot oder Verschlechterung des Allgemeinzustands gab eine Beschwerdedauer von länger als einer Woche vor Aufsuchen der Notaufnahme an.

\section{Verbleib nach Vorstellung in der Notaufnahme}

Von den 2090 Patienten mit Angaben zur Weiterversorgung nach Vorstellung in der ZNA wurden 1843 Patienten $(88,2 \%)$ ambulant belassen. 117 Patienten (5,6\%) wurden stationär aufgenommen oder in ein anderes Krankenhaus zur Aufnahme verlegt. 91 Patienten $(4,4 \%)$ wurden initial der Allgemeinmedizin zugeordnet, im weiteren Versorgungsverlauf jedoch an eine andere Fachabteilung innerhalb der ZNA abgegeben. Hier war dann der weitere Verbleib - ob ambulant oder stationär unklar. Insgesamt 34 Patienten (1,5\%) verließen die ZNA noch vor dem ersten Arztkontakt, vor dem abschließenden Arztgespräch oder gegen ärztlichen Rat. 


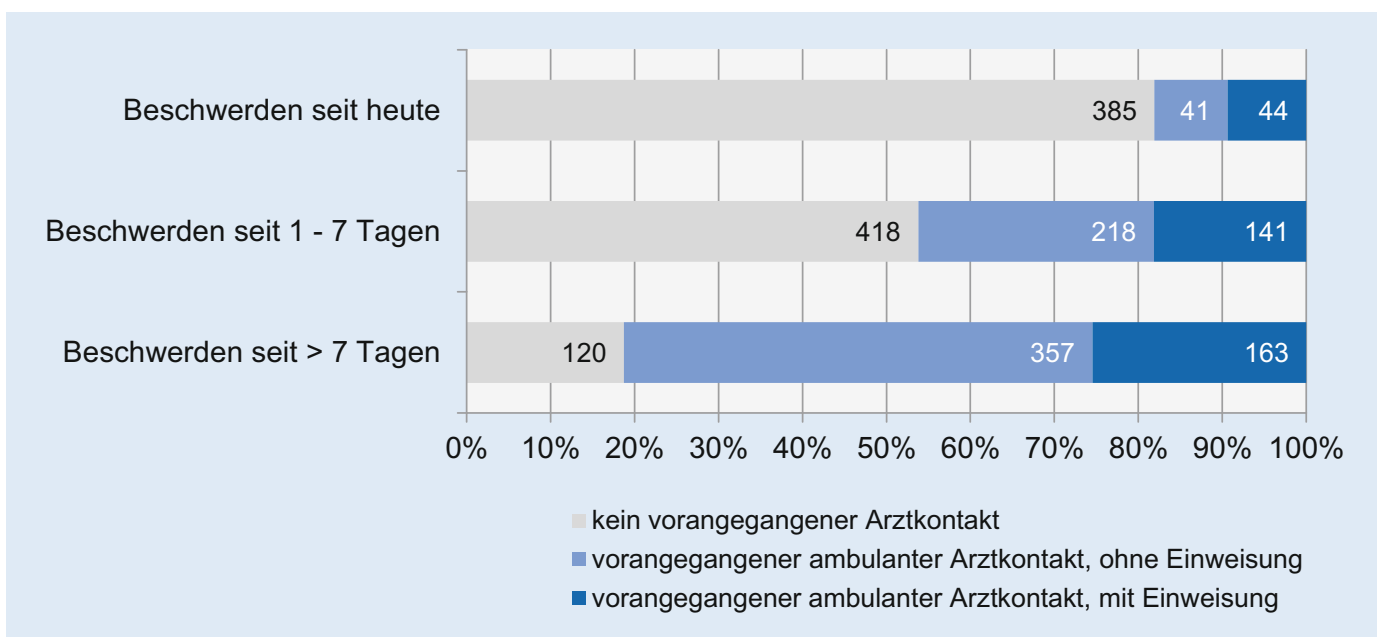

Abb. $2 \triangleleft$ Beschwerdedauer und vorangegangene ambulante Arztkontakte ${ }^{\mathrm{a}}$ $(n=1887) .{ }^{\text {a Hausarzt, Fach- }}$ spezialist, ärztlicher Bereitschaftsdienst, Notaufnahme

306 (87,7\%) der 349 Patienten, die mit ärztlicher Einweisung vorstellig wurden, konnten nach Diagnostik und Erstversorgung in die ambulante Versorgung entlassen werden, von den 1557 Selbstzuweisern blieben 1457 (93,6\%) ambulant. Differenziert nach den Top-20-Beratungsanlässen (- Tab. 1) zeigt sich, dass Patienten mit Fieber oder mit Verschlechterung des Allgemeinzustands mit jeweils $17,0 \%$ anteilig am häufigsten stationär aufgenommen wurden. Patienten mit Übelkeit und/oder Erbrechen als Vorstellungsgrund blieben am häufigsten ambulant.

\section{Diskussion}

Allgemeinmedizinisch versorgte Patienten sind Patienten, die mit niedriger Behandlungsdringlichkeit in der Notaufnahme der $\mathrm{MHH}$ vorstellig werden. Dennoch zeigt sich auch innerhalb dieser Subgruppe eine große Heterogenität bezüglich Beratungsanlässen, vorangegangener Arztkontakte und Beschwerdedauer vor dem Aufsuchen der Notaufnahme.

Die überwiegende Mehrheit dieser Patienten stellt sich mit gesundheitlichen Problemen vor, die seit mindestens dem Vortag bestehen, bei einem Drittel bereits seit mehr als einer Woche. Mehrere Querschnittsstudien zu nichtdringlichen Patientenkollektiven in Notaufnahmen, jedoch ohne Differenzierung nach versorgender Fachdisziplin, zeichnen ein ähnliches Bild. So wird von $35 \%$ der Patienten mit einer Beschwerdedauer von mindestens 3 Tagen [15], bei $23 \%$ bzw. $27 \%$ der Patienten von einem Auftreten der Beschwerden mehr als eine Woche vor Aufsuchen der Notaufnahme berichtet $[1,13]$.

Etwa die Hälfte der Patienten hat die ZNA der MHH als erste Anlaufstelle gewählt, während die andere Hälfte der Patienten zuvor versucht hatte, die Probleme in Arztpraxen, beim kassenärztlichen Bereitschaftsdienst oder in anderen Notaufnahmen zu lösen. Wir konnten zeigen, dass mit zunehmender Dauer der Beschwerden anteilig mehr Patienten bereits im ambulanten System ärztliche Hilfe in Anspruch genommen hatten. Zugangsbarrieren zur ambulanten Versorgung sowie pragmatische Gründe, von verschiedenen Autoren als Hauptmotive nichtdringlicher Patienten bzw. Selbstzuweiser für das direkte Aufsuchen der Notaufnahmen identifiziert $[7,13,18,19]$, scheinen für unser Patientenkollektiv zumindest für den Erstkontakt zur hausärztlichen Versorgung eine weniger große Rolle zu spielen.

Die einzelnen Beratungsanlässe der allgemeinmedizinischen ZNA-Patienten weisen unterschiedliche Anteile an Selbstzuweisern auf, die Beschwerdedauer, bevor die Notaufnahme aufgesucht wurde, variiert. Dies deutet darauf hin, dass bestimmte Motive jeweils abhängig vom zugrunde liegenden Gesundheitsproblem in den Vordergrund treten. Unter den Patienten, die zum Ausschluss einer Thrombose vorstellig werden, gibt es einen hohen Anteil an Vorabkontakten in der ambulanten Regelversorgung, bei denen häufig die Indikation zur Einweisung gestellt wurde, ein möglicher Hinweis, dass für die weitere Abklärung (Duplexsonographie) Zugangs- barrieren zur ambulanten fachspezialistischen Versorgung bestehen.

Patienten können die Entscheidung für eine bestimmte Anlaufstelle innerhalb des Gesundheitssystems nur symptombasiert treffen [13]. Das direkte Aufsuchen bei bestimmten Beschwerden könnte darauf hinweisen, dass diese Beschwerden hinsichtlich ihrer Behandlungsdringlichkeit schlecht eingeschätzt werden können und die Angst um die Gesundheit im Vordergrund steht. Beratungsanlässe, für die überdurchschnittlich häufig vor dem Aufsuchen der Notaufnahme bereits ärztliche Hilfe in Anspruch genommen wurde, könnten hingegen aufzeigen, dass sich die betroffenen Patienten im ambulanten System mit ihrem Gesundheitsproblem nicht hinreichend und sicher versorgt fühlen.

Eine nichtdringliche Ersteinschätzung ist nicht zwangsläufig gleichbedeutend mit einer "falschen" Vorstellung in der Notaufnahme. So weisen Notaufnahmepatienten trotz initialer Einstufung in die niedrigsten Dringlichkeitskategorien stationäre Aufnahmequoten zwischen 14 und $30 \%$ auf $[22,24]$. Wenngleich der Anteil stationärer Aufnahmen in unserem Patientenkollektiv für die Selbstzuweiser deutlich niedriger liegt, haben diese als nichtdringlich eingestuften Patienten dennoch aus der Rückschau betrachtet die richtige Anlaufstelle gewählt.

Hinsichtlich Art und Verteilung der Beratungsanlässe wird deutlich, dass allgemeinmedizinisch versorgte ZNA-Patienten nicht mit dem Patientenkollektiv einer Hausarztpraxis gleichgesetzt werden 


\begin{tabular}{|c|c|c|c|c|c|c|c|c|c|c|c|c|c|c|c|c|c|c|c|c|c|}
\hline 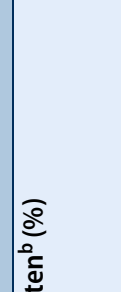 & 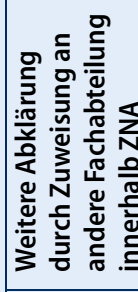 & & $\stackrel{\Rightarrow}{\nexists}$ & in & & $\equiv$ & 乎 & $\stackrel{\sim}{\sim}$ & $\stackrel{n}{\sim}$ & $\bar{\infty}$ & $\therefore$ & f & $\stackrel{\infty}{=}$ & 8 & $\bar{i}$ & $\stackrel{\circ}{\circ}$ & & : & ઝુ & $\stackrel{\circ}{m}$ & \\
\hline 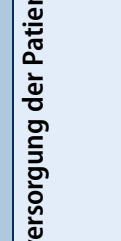 & 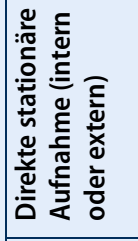 & : & $\stackrel{\sim}{m}$ & $\therefore$ & $\stackrel{O}{m}$ & $\bar{i}$ & in & $\stackrel{\sim}{\sim}$ & in & $\stackrel{5}{=}$ & $\stackrel{\circ}{\sim}$ & $\approx$ & $\stackrel{\circ}{m}$ & \& & $\stackrel{0}{i}$ & 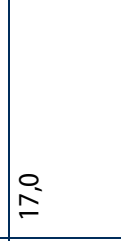 & $\stackrel{\infty}{\sim}$ & $\stackrel{\grave{N}}{\mathbf{N}}$ & 8 & $\stackrel{0}{m}$ & $\stackrel{8}{8}$ \\
\hline : & 旁 & $\frac{d}{\infty}$ & $\hat{\alpha}$ & $\hat{\bar{a}}$ & $\bar{\infty}$ & ऊू & $\underset{\infty}{\infty}$ & $\stackrel{\sim}{n}$ & $\begin{array}{l}\stackrel{\alpha}{\alpha} \\
\infty\end{array}$ & $\left.\begin{array}{c}\infty \\
\hdashline \\
\infty \\
\infty\end{array}\right)$ & $\frac{a}{\infty}$ & 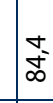 & హे & 号 & $\mid \begin{array}{l}0 \\
0 \\
0\end{array}$ & $\stackrel{\tilde{Q}}{\check{R}}$ & $\frac{d}{d}$ & $\frac{m}{2}$ & $\begin{array}{l}+ \\
⿱ 亠 凶 \\
\infty\end{array}$ & 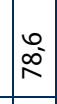 & \\
\hline$\frac{\sqrt{2}}{\frac{2}{2}}$ & 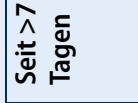 & 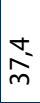 & 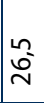 & $\underset{\sim}{\infty}$ & $\underset{\Im}{q}$ & $\stackrel{m}{i}$ & ๙ૂ & $\frac{n}{m}$ & $\approx$ & ind & $\stackrel{\simeq}{\sim}$ & $\bar{n}$ & ఓે & $\begin{array}{l}0 \\
\infty \\
m\end{array}$ & $\frac{m}{i}$ & 柋 & $\frac{\mathfrak{N}}{\tilde{F}}$ & $\stackrel{m}{\mp}$ & 雍 & $\bar{m}$ & \\
\hline 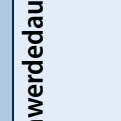 & 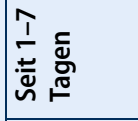 & s. & $\hat{i}$ & $\begin{array}{l}m \\
g \\
q\end{array}$ & 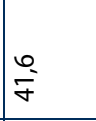 & 胥 & $\stackrel{\mathscr{I}}{\stackrel{I}{I}}$ & $\stackrel{n}{n}$ & f & $\begin{array}{l}a \\
D_{0}^{+} \\
\sim\end{array}$ & $\begin{array}{l}n \\
0 \\
0\end{array}$ & $\stackrel{\substack{j \\
m}}{ }$ & ì & 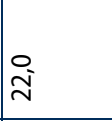 & $\mid \begin{array}{l}0 \\
0 \\
0\end{array}$ & 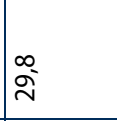 & 亲 & $\stackrel{a}{\sim}$ & $\begin{array}{l}\stackrel{0}{\sigma} \\
\dot{q}\end{array}$ & $\stackrel{\infty}{\infty}_{\substack{\infty \\
\sim}}$ & \\
\hline 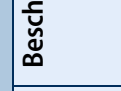 & $\frac{5}{\frac{5}{4}}$ & $\mathrm{n}^{2}$ & $\widehat{\infty}_{\infty}^{-}$ & $\stackrel{m}{\sim}$ & in & 孛 & $\stackrel{0}{i}$ & $\stackrel{m}{i}$ & 蒋 & $\stackrel{\infty}{m}$ & 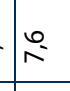 & ন & $\stackrel{+}{\stackrel{\sim}{*}}$ & 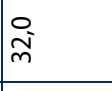 & $\mid \begin{array}{l}0 \\
0 \\
0\end{array}$ & $\stackrel{2}{\infty}$ & in & $\underset{\infty}{\stackrel{\infty}{~}}$ & $\begin{array}{l}\infty \\
\infty \\
\stackrel{\infty}{-\infty}\end{array}$ & $\overline{\tilde{m}}$ & \\
\hline 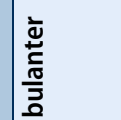 & 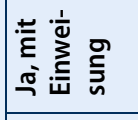 & ì & 吉 & $\stackrel{ \pm}{\Xi}$ & & $\stackrel{\infty}{\underset{I}{\Im}}$ & $\bar{\Xi}$ & $\infty_{\infty}^{\infty}$ & 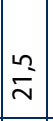 & $\tilde{I}$ & {$\left[\begin{array}{l}0 \\
\dot{f}\end{array}\right.$} & $\cong$ & $\stackrel{m}{f}$ & 践 & $\mid \begin{array}{c}\dot{m} \\
\tilde{n}\end{array}$ & 吕 & $\infty_{\infty}^{m}$ & $\stackrel{\Xi}{=}$ & ñ. & 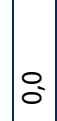 & \\
\hline 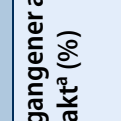 & 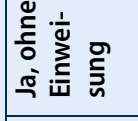 & $\bar{m}$ & $\mid \begin{array}{l}0 \\
\frac{0}{m}\end{array}$ & $\begin{array}{l}\infty \\
\vdots \\
\vdots\end{array}$ & & $\stackrel{m}{i}$ & $\stackrel{m}{s}$ & $\ddot{\dot{a}}$ & 足 & $\bar{m}$ & $\hat{\sigma}$ & : & ì & 吕 & $\left(\begin{array}{l}0 \\
i \\
i\end{array}\right.$ & $\hat{n}$ & $\stackrel{\bar{\sigma}}{\check{\gamma}}$ & $\bar{\AA}$ & $\frac{m}{m}$ & 吕 & 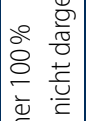 \\
\hline 漚 & $\frac{c}{\frac{c}{\bar{v}}}$ & $\bar{g}$ & 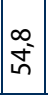 & $\overline{i n}$ & $\underset{f}{\stackrel{g}{F}}$ & 袁 & 今े & $\stackrel{n}{\sigma}$ & $\overline{g o s}$ & 㝵 & $\underset{\mathcal{N}}{\mathcal{f}}$ & ले & $\stackrel{\infty}{i=}$ & 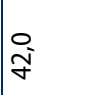 & 每 & 圌 & $\overline{\bar{m}}$ & $\begin{array}{l}\mathscr{0} \\
\stackrel{\infty}{o}\end{array}$ & 品 & 品 & 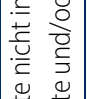 \\
\hline 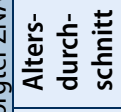 & 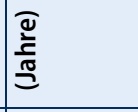 & $\hat{\sigma}$ & 㞼 & $\stackrel{n}{2}$ & 兑 & 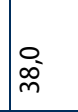 & $\stackrel{t}{i n}$ & $\hat{\sigma}$ & $\begin{array}{l}\infty \\
\infty \\
n^{0}\end{array}$ & $\stackrel{m}{i n}$ & $\overline{\bar{N}}$ & $\tilde{\dot{\sigma}}$ & $\begin{array}{l}\infty \\
\infty \\
m \\
m\end{array}$ & $\frac{0}{\dot{q}}$ & 商 & fo & $\underset{\tilde{\gamma}}{\tilde{\gamma}}$ & $\begin{array}{l}0 \\
\text { ò }\end{array}$ & $\begin{array}{l}0 \\
\text { in } \\
\text { in }\end{array}$ & $\bar{z}$ & 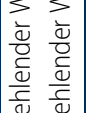 \\
\hline 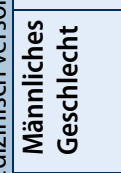 & $\stackrel{\bar{\varrho}}{ }$ & $\tilde{q}$ & gे & \begin{tabular}{l}
\multirow{0}{0}{} \\
in
\end{tabular} & $\begin{array}{l}0 \\
\dot{a}\end{array}$ & 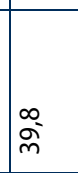 & $\widehat{o}$ & ì & $\mid \begin{array}{c}\infty \\
\cdots \\
m\end{array}$ & $\begin{array}{l}\infty \\
\tilde{m}^{-}\end{array}$ & $\overline{\dot{y}}$ & 离 & $\begin{array}{l}\text { Na } \\
\stackrel{\alpha}{q}\end{array}$ & 絮 & 吕 & fo & in & 过 & is & : & 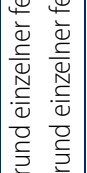 \\
\hline 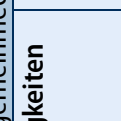 & 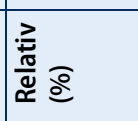 & & & $\overline{\text { in }}$ & f & 事 & $\hat{m}$ & $\underset{m}{\dot{m}}$ & $m_{m}^{m}$ & $\bar{m}=$ & $\stackrel{\infty}{i}$ & $\hat{i}$ & 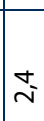 & $\bar{i}$ & $\stackrel{i}{i}$ & i & & $\stackrel{2}{\cong}$ & $\Xi$ & $\cong$ & $\frac{\pi}{0} \frac{\pi}{0}$ \\
\hline 产 & 嵩 & & & $\bar{\beth}$ & 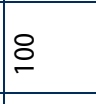 & बू & $\widehat{\infty}$ & $\infty$ & 2 & I & ๑ & t & i & in & f & f & $\stackrel{m}{\circ}$ & $\tilde{m}$ & $\tilde{m}$ & $\stackrel{\infty}{\sim}$ & \\
\hline & 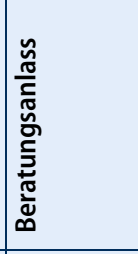 & ह气 & & 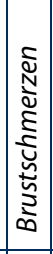 & 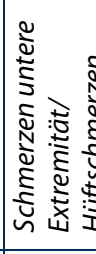 & 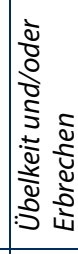 & 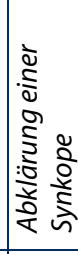 & 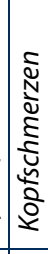 & 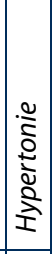 & 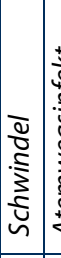 & 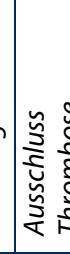 & 产 & & 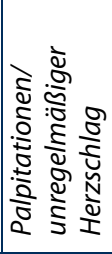 & 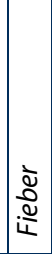 & 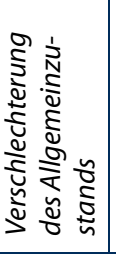 & 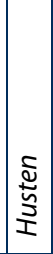 & 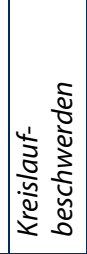 & 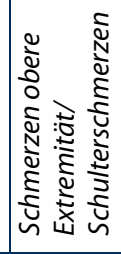 & 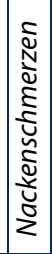 & 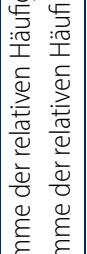 \\
\hline & 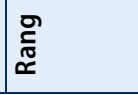 & & & & & in & & $\wedge$ & & & & & & & $\cong$ & & & & g & 尺 & \\
\hline
\end{tabular}


können. So dominiert in der hausärztlichen Versorgung der Beratungsanlass Husten, gefolgt von Rücken- und Nackenbeschwerden. Bauchschmerzen hingegen liegen mit 2\% aller Beratungsanlässe auf dem neunten Rang [8]. Bei den allgemeinmedizinischen ZNA-Patienten dominiert hingegen der Beratungsanlass Bauchschmerzen mit einem Anteil von knapp $14 \%$. Brustschmerzen und die Abklärung einer Synkope, bei den ZNA-Patienten unter den zehn häufigsten Beratungsanlässen, liegen in der hausärztlichen Praxis nicht innerhalb der ersten 20 Ränge [8]. In einer Studie zu Fallkosten ambulanter Notaufnahmepatienten wurden als teuerste Leitsymptome neben Bauchschmerzen die Beschwerden Kopfschmerzen, Schwindel, Synkope, Palpitation, Brustschmerz und Übelkeit identifiziert, da diese auf schwere Ereignisse hindeuten können und deshalb umfangreiche diagnostische Maßnahmen nach sich ziehen [10]. Sechs der sieben teuersten Leitsymptome befinden sich unter den zehn häufigsten Beratungsanlässen allgemeinmedizinisch versorgter ZNA-Patienten, sie machen damit in Summe 35\% aller dokumentierten Beratungsanlässe aus. Die universitäre Notaufnahme ist im Gegensatz zur Hausarztpraxis kein Niedrigprävalenzbereich [26], zudem kann in der Entscheidungsfindung bei unbekannten Patienten weder auf die „erlebte Anamnese“ zurückgegriffen noch das „abwartende Offenhalten " in der ZNA praktiziert werden [16]. Allgemeinmedizin in der zentralen Notaufnahme unterliegt somit gänzlich anderen medizinischen und sozialen Rahmenbedingungen als Allgemeinmedizin in der Hausarztpraxis.

\section{Limitationen}

Hinsichtlich der Einordnung der Ergebnisse und deren Übertragbarkeit sind folgende Limitationen zur berücksichtigen:

- Die Ergebnisse beziehen sich auf die zur Fachdisziplin Allgemeinmedizin triagierten Notfallpatienten einer universitären Notaufnahme. Diese Subgruppe wird annähernd vollständig abgebildet, sie spiegelt jedoch nur einen Teil der nichtdringlichen Patienten wider. Behandlungsfälle an Wochenenden sowie im Zeitraum von 18-8 Uhr wurden nicht in die Betrachtung einbezogen.

- Im Zugang zur Notaufnahme wurde zwischen Patienten mit und ohne ärztliche Einweisung unterschieden, der Zugang über den Rettungsdienst dabei nicht gesondert ausgewiesen.

\section{Ausblick}

Angesichts der Debatte um die ungerechtfertigte Inanspruchnahme der Notfallversorgung bietet die Integration der Allgemeinmedizin in die Notaufnahme den Vorteil, allgemeinmedizinische Versorgung für Patienten mit niedriger Behandlungsdringlichkeit vorhalten zu können, ohne im Bedarfsfall auf diagnostische und therapeutische Ressourcen eines Krankenhauses verzichten zu müssen.

\section{Fazit für die Praxis}

- Die Notaufnahme scheint von vielen Patienten als Möglichkeit genutzt zu werden, zusätzlich zur (vertrags-)ärztlichen Versorgung ärztliche Hilfe auch bei schon länger bestehenden Beschwerden zu erhalten.

- Auch bei initial nichtdringlich eingeschätzten Patienten mit Beschwerden aus dem allgemeinmedizinischen Spektrum kann eine stationäre Aufnahme indiziert sein.

- Krankenhäuser und Arztpraxen haben unter den gegebenen Rahmenbedingungen nur sehr begrenzte Möglichkeiten zur Steuerung des Patientenverhaltens.

Korrespondenzadresse

\section{Tanja Schleef}

Institut für Allgemeinmedizin, Medizinische Hochschule Hannover

Carl-Neuberg-Str. 1, 30625 Hannover, Deutschland

schleef.tanja@mh-hannover.de

Funding. Open Access funding enabled and organized by Projekt DEAL.

\section{Einhaltung ethischer Richtlinien}

Interessenkonflikt. T. Schleef, N. Schneider und $O$. Krause geben an, dass kein Interessenkonflikt besteht.

Für diesen Beitrag wurden von den Autoren keine Studien an Menschen oder Tieren durchgeführt.
Für die aufgeführten Studien gelten die jeweils dort angegebenen ethischen Richtlinien.

Open Access. Dieser Artikel wird unter der Creative Commons Namensnennung 4.0 International Lizenz veröffentlicht, welche die Nutzung, Vervielfältigung, Bearbeitung, Verbreitung und Wiedergabe in jeglichem Medium und Format erlaubt, sofern Sie den/die ursprünglichen Autor(en) und die Quelle ordnungsgemäß nennen, einen Link zur Creative Commons Lizenz beifügen und angeben, ob Änderungen vorgenommen wurden.

Die in diesem Artikel enthaltenen Bilder und sonstiges Drittmaterial unterliegen ebenfalls der genannten Creative Commons Lizenz, sofern sich aus der Abbildungslegende nichts anderes ergibt. Sofern das betreffende Material nicht unter der genannten Creative Commons Lizenz steht und die betreffende Handlung nicht nach gesetzlichen Vorschriften erlaubt ist, ist für die oben aufgeführten Weiterverwendungen des Materials die Einwilligung des jeweiligen Rechteinhabers einzuholen.

Weitere Details zur Lizenz entnehmen Sie bitte der Lizenzinformation aufhttp://creativecommons.org/ licenses/by/4.0/deed.de.

\section{Literatur}

1. Diserens L, Egli L, Fustinoni $S$ et al (2015) Emergency department visits for non-life-threatening conditions: evolution over 13 years in a Swiss urban teaching hospital. Swiss Med Wkly 145:w14123

2. Dräther H, Schäfer T (2017) Die ambulante Notfallversorgung in Notfallambulanzen und bei Vertragsärzten im Zeitraum 2009 bis 2014. In: Klauber J, Geraedts M, Friedrich J, Wasern J (Hrsg) Krankenhaus-Report 2017. Schattauer, Stuttgart, S 25-38

3. Geissler A, Quentin W, Busse R (2017) Umgestaltung der Notfallversorgung: Internationale Erfahrungen und Potenziale für Deutschland. In: Klauber J, Geraedts M, Friedrich J, Wasem J (Hrsg) Krankenhaus-Report 2017. Schattauer, Stuttgart, S 41-59

4. Haas C, Larbig M, Schöpke Tet al (2015) Gutachten zur ambulanten Notfallversorgung im Krankenhaus. Fallkostenkalkulation und Strukturanalyse, S1-82

5. Holzinger F, Oslislo S, Mockel M et al (2020) Selfreferred walk-in patients in the emergency department- who and why? Consultation determinants in a multicenter study of respiratory patients in Berlin, Germany. BMC Health Serv Res 20:848

6. Köster C, Wrede S, Herrmann T et al (2016) Ambulante Notfallversorgung. Analyse und Handlungsempfehlungen. AQUA-Institut für angewandte Qualitätsförderung und Forschung im Gesundheitswesen $\mathrm{GmbH}$, Göttingen

7. KraaijvangerN, RijpsmaD, WillinkLetal (2017) Why patients self-refer to the emergency department: a qualitative interview study. J Eval Clin Pract 23:593-598

8. Kühlein T, Laux G, Gutscher A et al (2008) Kontinuierliche Morbiditätsregistrierung in der Hausarztpraxis. Vom Beratungsanlass zum Beratungsergebnis. Urban \& Vogel, München

9. Malmström $T$, Huuskonen $O$, Torkki $P$ et al (2012) Structured classification for ED presenting complaints-From free text field-based approach 
to ICPC-2 ED application. Scand J Trauma ResusC Emerg Med 20:76

10. Meier F, Bauer K, Schöffski O et al (2015) Zur Ökonomie ambulanter Notaufnahmepatienten. Notfall Rettungsmed 19:33-40

11. Merguet $P$ (2021) Ambulante vs. stationäre Behandlung in der zentralen Notaufnahme. Notfall Rettungsmed. https://doi.org/10.1007/s10049021-00839-3

12. Pines JM, Hilton JA, Weber EJ et al (2011) International perspectives on emergency department crowding. Acad Emerg Med 18:1358-1370

13. Reinhold A, Greiner F, Schirrmeister W et al (2020) Der Notfall "geht" ins Krankenhaus. Med Klin Intensivmed Notfmed. https://doi.org/10.1007/ s00063-020-00681-4

14. Ries M, Christ M (2015) Qualitätsmanagement in der Notaufnahme: Fehlende einheitliche Standards zur kennzahlenbasierten Steuerung. Med Klin Intensivmed Notfmed 110:589-596

15. Scherer M, Luhmann D, KazekA et al (2017) Patienten in Notfallambulanzen. Querschnittstudie zur subjektiv empfundenen Behandlungsdringlichkeit und zu den motiven, die Notfallambulanzen von Krankenhäusern aufzususchen. Dtsch Arztebl Int 114:645-652

16. Schleef T, Klindtworth $K$, Jünger $S$ et al (2016) „.... das ist da anders als das typische hausärztliche Handeln, dieses ,abwartende Offenhalten', das haben wir ja nicht". Allgemeinärzte in der Notaufnahme einer Universitätsklinik: eine qualitative Untersuchung.ZAllg Med 92:198-202

17. Schleef T, Schneider N, Tecklenburg A et al (2017) Allgemeinmedizin in einer universitären Notaufnahme - Konzept, Umsetzung und Evaluation. Gesundheitswesen 79:845-851

18. Schmiedhofer M, Mockel M, Slagman A et al (2016) Patient motives behind low-acuity visits to the emergency department in Germany. A qualitative study comparing urban and rural sites. BMJ Open 6:e13323

19. Schmiedhofer MH, Searle J, Slagman A et al (2017) Inanspruchnahme zentraler Notaufnahmen. Qualitative Erhebung der Motivation von Patientinnen und Patienten mit nichtdringlichem Behandlungsbedarf. Gesundheitswesen 79:835-844

20. Schöpke T, Dodt C, Brachmann M et al (2014) Statusbericht aus deutschen Notaufnahmen. Notfall Rettungsmed 17:660-670

21. Searle J, Muller R, Slagman A et al (2015) Überfüllung der Notaufnahme. Gründe und populationsbezogene Einflussfaktoren. Notfall Rettungsmed 18:306-315

22. Slagman A, GreinerF, Searle Jet al (2019) Suitability of the German version of the Manchester Triage System to redirect emergency department patients to general practitioner care: a prospective cohort study. BMJ Open 9:e24896

23. Somasundaram R, Geissler A, Leidel BA et al (2018) Beweggründe für die Inanspruchnahme von Notaufnahmen. ErgebnisseeinerPatientenbefragung. Gesundheitswesen 80:621-627

24. Van Der Wulp I, Schrijvers AJ, van Stel HF (2009) Predicting admission and mortality with the Emergency Severity Index and the Manchester Triage System: a retrospective observational study. Emerg Med J 26:506-509

25. Wahlster P, Czihal T, Gibis B et al (2020) Sektorenübergreifende Entwicklungen in der Notfallversorgung - Eine umfassende Analyse ambulanter und stationärer Notfälle von 2009 bis 2015. Gesundheitswesen 82:548-558

26. Wübken M, Oswald J, Schneider A (2013) Umgang mit diagnostischer Unsicherheit in der

\section{General practice in the emergency department-Which patients? Which complaints?}

Background: Because increasing numbers of patients remain in outpatient care after visiting the emergency department $(E D)$, general practitioners have been integrated into the ED of a university hospital. Within the ED, patients who present with general medical complaints and show low urgency for treatment in the initial assessment are assigned to the general practitioner. The aim of this study was to characterize these patients with regard to their health complaints that led to their presentation in the ED. Methods: Descriptive analysis based on routine data and a self-developed evaluation form.

Results: About $80 \%$ of the patients visited the ED without a physician's referral. The five most frequent reasons for consultation were abdominal pain, back pain, chest pain, pain in the lower extremities and nausea and/or vomiting. In almost $25 \%$ of the patients, the complaints started on the day of the presentation, in about $33 \%$ the health complaints had existed for more than a week. Half of the patients had already received outpatient medical treatment for their complaints; in the group of patients with complaints lasting longer than one week, this proportion was more than $80 \%$. Patients with fever or impaired general state of health were admitted to hospital most frequently. Of the self-referrals, almost $94 \%$ could be discharged to outpatient care. Conclusion: Patients visit the ED with nonacute complaints and despite previous outpatient treatment. The most frequent reasons for consultation of patients treated by general practitioners in the ED only partially overlap those of patients treated by general practitioners in regular outpatient care.

\section{Keywords}

University hospital · Outpatient care · Self-referrals · Presenting complaint · Health care research

Hausarztpraxis. Z Evid Fortbild Qual Gesundhwes

107:632-637 